\title{
Análise da representatividade e da densidade aparente como estimadoras do módulo de elasticidade da classe C60 da NBR7190:1997
}

\author{
Representativeness analysis and apparent density as \\ estimators of modulus of elasticity of class C60 of \\ NBR7190:1997
}

${ }^{1}$ Francisco Antonio Rocco Lahr 1 Universidade de São Paulo São Carlos - SP - Brasil

${ }^{2}$ Eduardo Chahud 2Universidade Federal de Minas Gerais Belo Horizonte - MG - Brasil

${ }^{3}$ Felipe Nascimento Arroyo 3Universidade Federal de São Carlos São Carlos - SP - Brasil ${ }^{4}$ André Luis Christofro
${ }_{4}$ Universidade Federal de São Carlos
São Carlos - SP - Brasil

${ }^{5}$ Edson Fernando Castanheira Rodrigues ${ }^{5}$ Universidade Federal de São Carlos São Carlos - SP - Brasil

${ }^{6}$ João Paulo Boff Almeida ${ }^{6}$ Universidade Federal de São Carlos São Carlos - SP - Brasil

${ }^{7}$ Vinicius Borges de Moura Aquino ${ }^{7}$ Universidade Federal do Sul e Sudeste Santana do Araguaia - PA - Brasil

Recebido em 20/10/20 Aceito em 07/07/21

\section{Francisco Antonio Rocco Lahr \\ Eduardo Chahud \\ Felipe Nascimento Arroyo \\ André Luis Christoforo \\ Edson Fernando Castanheira Rodrigues \\ João Paulo Boff Almeida \\ Vinicius Borges de Moura Aquino}

\section{Resumo}

$\mathbf{P}$

ara o dimensionamento correto de estruturas de madeira, as premissas da norma brasileira são de extrema valia como norte para os engenheiros estruturais, pois exibem valores padrão de resistência. Dessa forma, este estudo tem como objetivo analisar se o valor do módulo de elasticidade da classe C60 exibido na norma brasileira confere com os valores obtidos em ensaio de 23 espécies brasileiras, utilizando a metodologia Bootstrap com 1 milhão de simulações. Além disso, analisa-se, com modelos de regressão, se é possível estimar a propriedade de rigidez $\left(E_{c 0}\right)$ dessas 23 espécies em função da densidade aparente. É possível concluir que a norma brasileira superestima o módulo de elasticidade das madeiras brasileiras, apresentado um valor quase $35 \%$ superior ao que realmente elas resistem, evidenciando que a norma necessita passar por revisão. Outro ponto é que os modelos de regressão não mostram significância, ou seja, com a densidade aparente não é possível estimar a propriedade de rigidez analisada.

Palavras-chave: Classe de resistência. Densidade aparente. Rigidez. Madeira.

\section{Abstract}

For correct dimensioning of wood structures, the premises of the Brazilian standard are extremely valuable as a guide for structural engineers, as it exhibits standard values of resistance. Thus, this study aims to analyze whether the value of the class C60 elasticity module shown in the Brazilian standard matches the values obtained in the test of 23 Brazilian species, using the Bootstrap methodology with 1 million simulations. In addition, it sought to analyze, with regression models, whether it is possible to estimate this rigidity property $\left(E_{c 0}\right)$ of these 23 species as a function of apparent density. Finally, it was possible to conclude that the Brazilian standard overestimates the modulus of elasticity of Brazilian woods, presenting a value almost $35 \%$ higher than what actually resists, showing that the standard needs to be revised. Another point was that the regression models do not show significance, in other words, with the apparent density it was not possible to estimate the analyzed rigidity property.

Keywords: Strength class. Apparent density. Stiffness. Wood.

LAHR, F. A. R.; CHAHUD, E.; ARROYO, F. N.; CHRISTOFORO, A. L.; RODRIGUES, E. F. C.; ALMEIDA, J. P.B.; AQUINO, V. B. de M. Análise da representatividade e da densidade aparente como estimadoras do módulo de elasticidade da classe C60 da NBR7190:1997. Ambiente Construído, Porto Alegre, v. 22, n. 1, p. 139-146, jan. /mar. 2022.

ISSN 1678-8621 Associação Nacional de Tecnologia do Ambiente Construído. http: //dx.doi.org/10.1590/s1678-86212022000100583 


\section{Introdução}

A madeira é um material de excelentes propriedades estruturais, principalmente por possuir resistência tanto à tração como à compressão. Outro ponto importante é sua fonte renovável, ao contrário das outras estruturas usualmente utilizadas, concreto armado e metálica. À vista disso, a madeira, quando feito o reflorestamento, pode ser considerada um dos melhores materiais para se utilizar em estruturas (PIGOZZO et al., 2018a, 2018b).

De acordo com Bacha (2017), “[...] o Brasil se destaca no cenário internacional por suas extensas florestas nativas tropicais e pelo plantio de florestas homogêneas com espécies exóticas [...]". Ainda segundo o autor, o Brasil possui 493,5 milhões de hectares de florestas, o equivalente a $12 \%$ do planeta. No Brasil, a NBR 7190 (ABNT, 1997) rege as premissas para o dimensionamento desse tipo de estrutura no Brasil. A Tabela 1 apresenta as classes de resistência com as respectivas propriedades físicas e mecânicas médias e características que podem ser tomadas com base do dimensionamento.

Para um dimensionamento correto e seguro de uma estrutura, é ideal que se conheçam todas as propriedades de resistência e rigidez (OLIVEIRA et al., 2019; PIGOZZO et al., 2018c). No caso da estrutura de madeira, vários ensaios seriam necessários para se obterem as propriedades, sendo alguns mais complicados de ser realizados. Contudo, existem algumas formas fáceis de obter as propriedades para realizar o dimensionamento. Uma delas é a utilização do método simplificado contido na NBR 7190, que consegue fazer a estimativa com a relação entre as propriedades. Outra é através da tabela de classes de resistência (Tabela 1). Por último, através de modelos de regressão, utilizando a densidade aparente como estimadora das outras propriedades (ADAMOPOULOS; PASSIALIS, 2010).

Entretanto, conforme estudo mostrado por Couto et al. (2020), a norma brasileira atual está desatualizada, e os valores exibidos podem não ser iguais à realidade. Em seu estudo, foi analisada a relação entre as propriedades (método simplificado) considerando dez espécies de madeira do grupo das folhosas, utilizando a análise estatística Bootstrap. Com isso, foi possível concluir que os valores analisados são 86,40\% superiores aos exibidos na norma brasileira (ABNT, 1997), apontando a lacuna existente dessa norma.

Conforme o trabalho desenvolvido por Christoforo et al. (2017), em que se analisou a densidade aparente como estimadora das outras propriedades da Calycophyllum multiflorum, foi possível concluir que, a partir do valor da densidade, se conseguem estimar quatro propriedades das catorze analisadas: módulo de elasticidade médio, compressão perpendicular às fibras, módulo de elasticidade na compressão perpendicular às fibras e compressão paralela às fibras. O maior coeficiente de variação obtido foi de $85,23 \%$, utilizando o modelo logarítmico.

À vista disso, este artigo tem como objetivo avaliar a representatividade do módulo de elasticidade à compressão paralela às fibras $\left(E_{c 0}\right)$ da classe $\mathrm{C} 60$ para 23 espécies do grupo das folhosas brasileiras. O estudo analisou se o valor apresentado de $24.500 \mathrm{MPa}$ (Tabela 1) na norma brasileira de estruturas de madeira, a NBR 7190 (ABNT, 1997), está de acordo com a realidade, considerando um intervalo de confiança de 5\% e utilizando a técnica de reamostragem Bootstrap para simulação do intervalo de confiança médio (100 a 1.000.000 de simulações). Por fim, foi verificada a possibilidade de uso do valor da densidade aparente em estimar a resistência e o módulo de elasticidade à compressão paralela às fibras das espécies estudadas.

\section{Material e métodos}

As 23 espécies utilizadas neste trabalho são referenciadas no Quadro 1. Ressalta-se que todas essas espécies fazem parte da classe C60, conforme a NBR 7190 (ABNT, 1997), por possuírem $f_{c 0, k}$ igual ou superior a 60 $\mathrm{MPa}$.

As madeiras provenientes de lotes homogêneos foram devidamente estocadas, tendo resultado em teor de umidade próximo de 12\%, conforme as premissas da NBR 7190 (ABNT, 1997). Os métodos de ensaio da norma brasileira também foram seguidos para as obtenções das propriedades físicas e mecânicas.

Ainda, como recomenda a norma brasileira, foram fabricados e testados 12 corpos de prova por espécie para determinar o módulo de elasticidade na compressão paralela às fibras (Figura 1), assim com outros 12 para a determinação dos valores da densidade aparente, resultando em 552 determinações experimentais ao todo.

140Lahr, F. A. R.; Chahud, E.; Arroyo, F. N.; Christoforo, A. L.; Rodrigues, E. F. C.; Almeida, J. P.B.; Aquino, V. B. de M. 
Tabela 1 - Classe de resistência de madeiras folhosas

\begin{tabular}{|c|c|c|c|c|}
\hline Classes & $f_{c 0, k}[\mathrm{MPa}]$ & $f_{v 0, k}[\mathrm{MPa}]$ & $E_{c 0, m}[\mathrm{MPa}]$ & $\rho_{12} \%\left[\mathrm{~kg} / \mathrm{m}^{3}\right]$ \\
\hline $\mathrm{C} 20$ & $20 \leq f_{c 0, k}<30$ & 4 & 9.500 & 650 \\
\hline $\mathrm{C} 30$ & $30 \leq f_{c 0, k}<40$ & 5 & 14.500 & 800 \\
\hline $\mathrm{C} 40$ & $40 \leq f_{c 0, k}<60$ & 6 & 19.500 & 950 \\
\hline C60 & $f_{c 0, k} \geq 60$ & 8 & 24.500 & 1.000 \\
\hline
\end{tabular}

Fonte: adaptada de ABNT (1997).

\section{Quadro 1 - Nomenclatura popular das madeiras folhosas utilizadas no estudo}

\begin{tabular}{|c|c|c|c|}
\hline Nome popular & Nome popular & Nome popular & Nome popular \\
\hline Cutiúba & Sucupira & Mandioqueira & Angelim-ferro \\
\hline Garrote & Tachi & Oiuchu & Angelim-vermelho \\
\hline Guajará & Quina rosa & Breu-vermelho & Roxinho \\
\hline Ipê & Tatajuba & Champanhe & Angico-preto \\
\hline Itaúba & Jatobá & Maçaranduba & \\
\hline
\end{tabular}

Figura 1 - (a) Ilustração dos corpos de prova padronizados pela norma (5x5x15 cm - NBR 7190 (ABNT, 1997)); e (b) aparelho utilizado no ensaio do módulo de elasticidade à compressão paralela às fibras

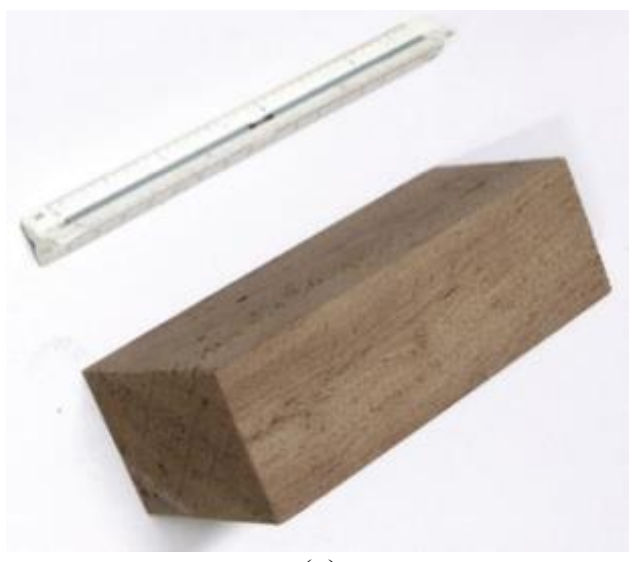

(a)

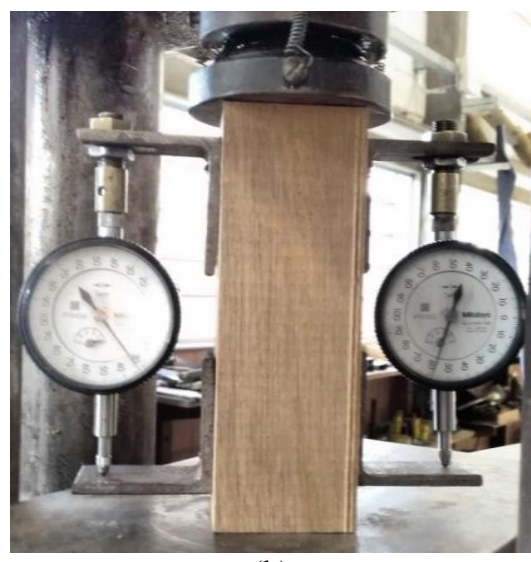

(b)

Os corpos de prova foram rompidos em máquina universal de ensaios (AMSLER - capacidade de carga de $25 \mathrm{t}$ ). $\mathrm{O}$ teor de umidade $(\mathrm{U})$ deles, no momento dos ensaios, foi obtido por meio do medidor de umidade por contato Marrari M5 (10,76 $\leq U \leq 12,96 \%)$. Com posse do teor de umidade das amostras, os valores de módulo de elasticidade foram corrigidos para o teor de umidade de 12\%, conforme indicado pela NBR 7190, com o auxílio da Equação 1, em que $E_{U}$ consiste na propriedade das amostras em relação ao teor de umidade $\mathrm{U}$, enquanto $E_{12 \%}$ consiste na propriedade a $12 \%$ de umidade.

$E_{12 \%}=E_{U} \cdot\left[1+\frac{2 \cdot(U-12)}{100}\right]$

Eq. 1

Com base nos valores corrigidos para $12 \%$ de umidade $\left(E_{12 \%}\right)$, a Equação 2 foi utilizada para a determinação do valor característico $\left(E_{c 0}\right)$ para a categorização das madeiras e análises propostas, em que $E_{1}, E_{2}$ a $E_{n}$ denotam os valores de módulo de elasticidade em ordem crescente dos 12 (n) corpos de prova testados, conforme a NBR 7190 (ABNT, 1997). Contudo, para a análise comparativa com a norma vigente, foi utilizado o valor médio das amostras, visto que a norma utiliza o médio, e não o característico.

$E_{c 0} \geq\left\{\begin{array}{c}E_{1} \\ 0.70 \cdot \frac{\sum_{i=1}^{n} E_{i}}{n} \\ 1.10 \cdot\left[2 \cdot\left(\frac{E_{1}+E_{2}+\cdots+E_{(n / 2)-1}}{(n / 2)-1}\right)-E n / 2\right]\end{array}\right.$ 


\section{Análise estatística: Bootstrap e T-test}

O intervalo de confiança médio (inferência de variação no valor médio da população com base na amostra), no nível de confiabilidade de $95 \%$, foi usado para calcular o valor médio, bem como o inferior $(2,5 \%)$ e superior $(97,5 \%)$, e o teste de Shapiro-Wilk (5\% de significância) foi utilizado para avaliar a distribuição normal dos valores de $E_{c 0}$ considerando o conjunto de 23 espécies de madeira. Para os pressupostos assumidos no teste de normalidade (T-test), o valor de $\mathrm{p}$ (probabilidade $\mathrm{p}$ ) igual ou superior ao nível de significância implica normalidade na distribuição $E_{c 0}$, que valida os resultados obtidos a partir do intervalo de confiança.

Para aumentar a confiabilidade dos resultados, foi utilizada a técnica de reamostragem Bootstrap para simular os intervalos de confiança médios ( $95 \%$ de confiabilidade), sendo consideradas de 100 a 1.000 .000 simulações (LAHR et al., 2014).

\section{Modelos de regressão - ANOVA}

O Quadro 2 mostra os modelos de regressão que foram utilizados nas estimativas. Nessa tabela, Y é a variável independente (módulo de elasticidade à compressão paralela às fibras $-E_{c 0}$ ), $\mathrm{X}$ a variável dependente (densidade aparente - $\rho_{a p}$ ) e "a", "b" e "b1" as constantes (parâmetros) das funções ajustadas pelo método dos mínimos quadrados. Pela ANOVA dos modelos de regressão a serem avaliados também ao nível de significância de $5 \%$, a hipótese nula estipulada consistirá na não representatividade dos modelos testados $(\mathrm{H} 0: \beta=0)$, e na representatividade como hipótese alternativa $(\mathrm{H} 1: \beta \neq 0)$. P-valor superior ao nível de significância considerado implica em aceitar H0 (o modelo testado não é representativo - variações de X são incapazes de explicar as variações em Y), refutando-a em caso contrário (o modelo testado é representativo). Além do uso da ANOVA, que permite aceitar ou não a representatividade dos modelos testados, os valores do coeficiente de determinação ajustado [R2(aj)] serão obtidos como forma de avaliar a capacidade das variações da variável independente $\mathrm{Y}$ em explicar a variável preditiva $\mathrm{X}$, possibilitando eleger, dentre os modelos considerados significativos, o de melhor ajuste por relação testada. Conforme Montgomery (2013), o coeficiente de determinação precisa ser superior à $70 \%$ para o modelo ser considerado significativo. (CHRISTOFORO et al., 2017).

\section{Resultados e discussão}

A Tabela 2 apresenta os valores médios da densidade aparente $\left(\rho_{12 \%}\right)$ e do módulo de elasticidade à compressão paralela às fibras médio $\left(E_{c 0, m}\right)$. Como dito anteriormente, todas as espécies são da classe C60 (Tabela 1).

A Tabela 3, a seguir, apresenta o coeficiente de variação $(C V)$ das propriedades analisadas, assim como o desvio padrão (DesvPad) e os valores mínimos (Mín.) e máximos (Máx.) obtidos nos ensaios. É possível notar que todas as propriedades ficaram com $C V$ abaixo dos $18 \%$, usualmente adotado pela norma NBR 7190 (ABNT, 1997), mostrando a qualidade dos ensaios laboratoriais e a homogeneidade dos lotes das peças.

\section{Análise estatística}

A Tabela 4, a seguir, representa o resultado do intervalo de confiança do $E_{c 0}$, considerando $95 \%$ de confiabilidade ( $5 \%$ de significância), para as médias das espécies da Tabela 2. Esses resultados são expressos através do método T-test com uma amostra e através do Bootstrap, considerando até 1.000.000 de simulações.

Quadro 2 - Modelos de regressão utilizados

\begin{tabular}{|l|c|}
\hline \multicolumn{1}{|c|}{ Ajuste } & Função $[\boldsymbol{Y = f ( X ) ]}$ \\
\hline Linear [Lin] & $Y=a+b 1 \cdot X$ \\
\hline Exponencial [Exp] & $Y=a \cdot e^{b 1 \cdot X}$ \\
\hline Logarítmico [Log] & $Y=a+b 1 \cdot \operatorname{Ln}(X)$ \\
\hline Geométrico [Geo] & $Y=a \cdot X^{b 1}$ \\
\hline
\end{tabular}

142Lahr, F. A. R.; Chahud, E.; Arroyo, F. N.; Christoforo, A. L.; Rodrigues, E. F. C.; Almeida, J. P.B.; Aquino, V. B. de M. 
Tabela 2 - Resultado das propriedades físicas e mecânicas médias das espécies analisadas

\begin{tabular}{l|c|c}
\hline \multicolumn{1}{c|}{ Espécie } & $\boldsymbol{\rho}_{\mathbf{1 2} \%}\left[\mathbf{k g} / \mathbf{m}^{\mathbf{3}}\right]$ & $\boldsymbol{E}_{\boldsymbol{c 0}, \boldsymbol{m}}[\mathbf{M P a}]$ \\
\hline Angelim-ferro 1 & 1.178 & 18.740 \\
Angelim-ferro 2 & 1.147 & 21.858 \\
Angelim-vermelho & 1.131 & 16.695 \\
Angico-preto & 898 & 19.880 \\
Breu-vermelho & 887 & 15.301 \\
Champanhe & 1.087 & 23.002 \\
Cutiúba & 1.160 & 18.591 \\
Garrote & 904 & 19.293 \\
Guajará & 781 & 14.489 \\
Ipê & 1.036 & 16.597 \\
Itaúba 1 & 915 & 17.443 \\
Itaúba 2 & 933 & 17.984 \\
Jatobá 1 & 1.082 & 21.403 \\
Jatobá 2 & 884 & 20.438 \\
Maçaranduba 1 & 1.143 & 21.900 \\
Maçaranduba 2 & 1.124 & 22.332 \\
Mandioqueira & 853 & 19.498 \\
Oiuchu & 932 & 17.770 \\
Quina Rosa & 935 & 22.483 \\
Roxinho & 938 & 21.080 \\
Sucupira & 1.106 & 21.484 \\
Tachi & 1.045 & 19.901 \\
Tatajuba & 935 & 18.238 \\
\hline
\end{tabular}

Tabela 3 - Resultado das propriedades analisadas

\begin{tabular}{c|c|c|c|c|c}
\hline Propriedades & Média & DesvPad & $\boldsymbol{C V}$ & Mín. & Máx. \\
\hline$\rho_{a p}[\mathrm{~kg} / \mathrm{m}]$ & $1.001,50$ & 117,70 & $11,75 \%$ & 781 & 1178 \\
$E_{c 0}[\mathrm{MPa}]$ & 19.409 & 2.366 & $12,19 \%$ & 11.489 & 23.002 \\
\hline
\end{tabular}

Tabela 4 - Resultado do intervalo de confiança para o módulo de elasticidade à compressão paralela às fibras (MPa)

\begin{tabular}{l|c|c|c}
\hline \multirow{2}{*}{ Método } & \multicolumn{3}{|c}{ CI (95\% de confiabilidade) } \\
\cline { 2 - 4 } & Limite inferior & Média & Limite superior \\
\hline T-test & $18.385,523$ & $19.408,696$ & $20.431,868$ \\
Bootstrap - 100 simulações & $18.502,880$ & $19.395,548$ & $20.183,346$ \\
Bootstrap - 500 simulações & $18.502,880$ & $19.431,535$ & $20.223,357$ \\
Bootstrap - 1.000 simulações & $18.412,642$ & $19.411,000$ & $20.200,372$ \\
Bootstrap - 5.000 simulações & $18.462,868$ & $19.398,901$ & $20.194,413$ \\
Bootstrap - 10.000 simulações & $18.426,263$ & $19.408,011$ & $20.203,777$ \\
Bootstrap - 50.000 simulações & $18.458,612$ & $19.411,616$ & $20.193,561$ \\
Bootstrap - 100.000 simulações & $18.452,653$ & $19.407,427$ & $20.197,818$ \\
Bootstrap - 500.000 simulações & $18.448,396$ & $19.408,403$ & $20.196,115$ \\
Bootstrap - 1.000.000 simulações & $18.450,950$ & $19.408,802$ & $20.193,561$ \\
\hline
\end{tabular}

Como se pode observar, o menor valor obtido em todas as análises estatísticas para a classe C60 foi de $18.450,95 \mathrm{MPa}$ de módulo de elasticidade à compressão paralela às fibras, enquanto a norma considera $\mathrm{o}$ valor de $24.500 \mathrm{MPa}$. Dessa forma, é possível notar que a NBR 7190 (ABNT, 1997) considera um valor superior $(32,78 \%)$ do que a madeira realmente possui de rigidez, fazendo com que a estrutura fique subdimensionada. Isso acarretaria um grande problema estrutural, visto que a estabilidade da estrutura, principalmente, estaria comprometida. 
Para um melhor entendimento do problema acarretado com esse erro da norma, se se considerar uma viga biapoiada (Figura 2), sujeita à ação de forças permanentes (g) e acidentais (q) distribuídas ao longo do vão $\mathrm{L}$, e medidas da seção transversal " $\mathrm{b}$ " e " $h$ " para a base e altura da seção transversal retangular respectivamente, é possível fazer a análise a seguir.

Para esse esquema adotado, o deslocamento máximo ocorre no meio do vão. A deformação máxima é determinada pela equação a seguir, conforme as premissas da NBR 7190 (ABNT, 1997).

$\delta_{\text {máx }}=\frac{5 \cdot(g+q) \cdot L^{4}}{32 \cdot E_{c 0} \cdot b \cdot h^{3}}$

Para a condição de ELU, a norma brasileira estabelece um limite de deslocamento vertical máximo de L/200 (estruturas correntes sem materiais frágeis não estruturais). Adotando os valores $b=10 \mathrm{~cm}, h=15 \mathrm{~cm}$, $L=350 \mathrm{~cm}$ e $E_{c 0}=24.500 \mathrm{MPa}$ (ABNT, 1997), o deslocamento-limite (L/200) é igual a $1,75 \mathrm{~cm}$. Para que não ultrapasse o deslocamento-limite, o máximo carregamento suportado por essa viga seria de 6,17 $\mathrm{kN} / \mathrm{m}(\mathrm{g}+\mathrm{q})$. Entretanto, considerando o módulo de elasticidade encontrado nesta pesquisa, de 18.450,95 $\mathrm{MPa}$, o máximo carregamento suportado para não ultrapassar a deformação máxima seria de 4,65 kN/m (24,64\% inferior). Caso a carga fosse concentrada, a diferença seria de $24,69 \%$. À vista disso, as estruturas de madeira estão sendo dimensionadas erroneamente, podendo ocorrer diversas patologias (ANDRADE JUNIOR et al., 2014).

\section{Modelos de regressão - ANOVA}

A Figura 3 mostra o melhor resultado do modelo de regressão obtido, utilizando a densidade aparente da classe estudada como estimadora do módulo de elasticidade à compressão paralela às fibras.

Através da Figura 3 é possível concluir que a densidade aparente não se apresentou como boa estimadora por apresentar coeficientes de determinação $\left(R^{2}\right)$ inferiores a $70 \%$, como indicado por Montgomery (2013), diferentemente da pesquisa apresentada por Christoforo et al. (2017) e Adamopoulos e Passialis (2010), em que o coeficiente de determinação chegou a $85,23 \%$ e a $92,30 \%$ respectivamente para o melhor ajuste. Uma forma de melhorar os ajustes obtidos, segundo Duarte et al. (2020), é considerando os constituintes químicos da madeira nos modelos de regressão.

Figura 2 - Estrutura considerada para a análise do efeito do módulo de elasticidade errôneo da norma

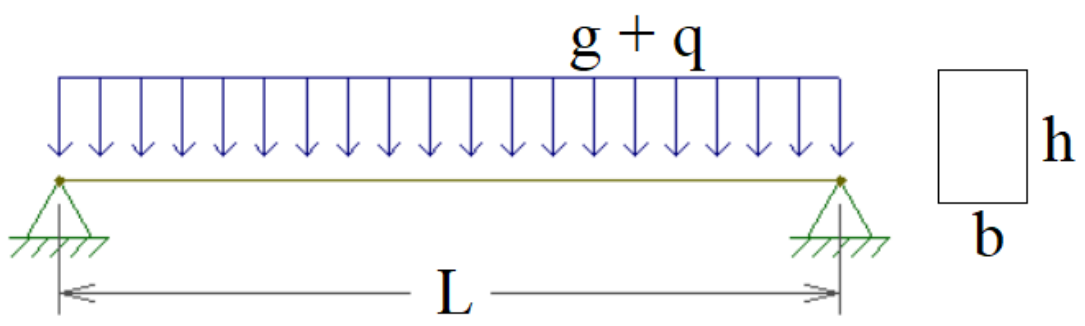

Figura 3 - Melhor ajuste obtido dentre os quatro modelos de regressão analisados

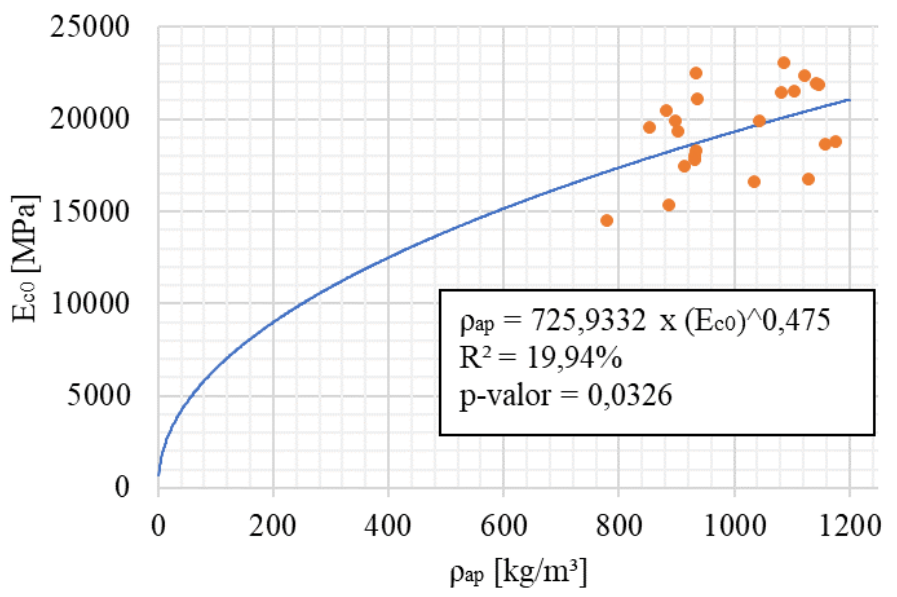

144Lahr, F. A. R.; Chahud, E.; Arroyo, F. N.; Christoforo, A. L.; Rodrigues, E. F. C.; Almeida, J. P.B.; Aquino, V. B. de M. 
Importante destacar que o p-valor foi inferior a 5\%; dessa forma, variações na densidade aparente são capazes de explicar as variações que ocorrem apenas no módulo de elasticidade na compressão paralela às fibras (modelo foi representativo).

\section{Considerações finais}

Com esta pesquisa, é possível chegar às conclusões a seguir:

(a) o coeficiente de variação foi inferior ao indicado pela norma brasileira de estruturas de madeira (18\%), evidenciando a qualidade nos ensaios laboratoriais executados e a homogeneidade das espécies utilizadas;

(b) o valor do módulo de elasticidade apresentado na norma brasileira de estruturas de madeira (24.500 $\mathrm{MPa}$ ) é muito superior (32,78\%) quando comparado com o módulo de elasticidade apresentado neste estudo $(18.450,95 \mathrm{MPa})$. À vista disso, a norma está subestimando as estruturas de madeira, podendo ocorrer vários tipos de patologia;

(c) a norma brasileira de estruturas de madeira precisa passar por uma revisão, visto que está subdimensionando as estruturas em relação ao módulo de elasticidade. Conforme visto no exemplo aplicado, a estrutura suportaria $24,64 \%$ menos carga do que a norma aceitaria;

(d) considerando os modelos de regressão analisados, o ajuste geométrico foi o que obteve melhor resultado, entretanto com a densidade aparente da classe C60 não foi possível estimar o módulo de elasticidade à compressão paralela às fibras, pois o ajuste não atingiu os $70 \%$ exigidos por Montgomery (2013); e

(e) a densidade aparente foi capaz de explicar as variações no módulo de elasticidade na compressão paralela às fibras, visto que o $\mathrm{p}$-valor foi inferior a $5 \%$.

\section{Referências}

ADAMOPOULOS, S.; PASSIALIS, C. Relationship of toughness and modulus of elasticity in static bending of small clear spruce wood specimens. European Journal of Wood and Wood Products, v. 68, p. 109-111, 2010.

ANDRADE JUNIOR, J. R. et al. Avaliação das estruturas de cobertura em madeira de um galpão de estoque de produtos químicos. Ambiente Construído, Porto Alegre, v. 14, n. 3, p. 75-85, jul./set. 2014.

ASSOCIAÇÃO BRASILEIRA DE NORMAS TÉCNICAS. NBR 7190: projeto de estruturas de madeira. Rio de Janeiro, 1997.

BACHA, C. J. C. Os mercados de produtos florestais no Brasil em 2017 e 2018. Esalq USP, 26 jun. 2017. Disponível em: https:/www.cepea.esalq.usp.br/br/opiniao-cepea/os-mercados-de-produtos-florestais-nobrasil-em-2017-e-2018.aspx. Acesso em: 9 out. 2020.

CHRISTOFORO, A. L. et al. Full characterization of Calycophyllum multiflorum wood specie. Engenharia Agrícola, v. 37, n. 4, p. 637-643, 2017.

COUTO, N. G. et al. Relação entre a resistência ao cisalhamento e a resistência à compressão paralela às fibras de madeiras folhosas. Ambiente Construído, Porto Alegre, v. 20, n. 4, p. 319-327, out./dez. 2020.

DUARTE, B. B. et al. Influence of physical and chemical components on the physical-mechanical properties of ten Brazilian wood species. Materials Research, v. 23, n. 2, p. 1-10, 2020.

LAHR, F. A. R. et al. Influência da temperatura de trabalho na resistência ao cisalhamento da madeira. In: CONGRESSO BRASILEIRO DE ENGENHARIA E CIÊNCIA DOS MATERIAIS, 21., Cuiabá, 2014.

Anais [...] Cuiabá, 2014.

MONTGOMERY, D. C. Design and analysis of experiments. 8. ed. Arizona: John Wiley \& Sons, 2013. Disponível em: http://faculty.business.utsa.edu/manderso/STA4723/readings/Douglas-C.-MontgomeryDesign-and-Analysis-of-Experiments-Wiley-2012.pdf. Acesso em: 9 out. 2020.

OLIVEIRA, G. O. B. et al. Technical feasibility study of the use of softwoods in lattice structure "howe" type for roofing (gaps between 8-18 meters). Current Journal of Applied Science and Technology, v. 35, n. 4 , p. $1-8,2019$. 
PIGOZZO, J. C. et al. Behavior of shear connectors formed by bonded-in "X" type steel bars in woodconcrete specimens. Current Journal of Applied Science and Technology, v. 28, n. 5, p. 1-8, 2018a.

PIGOZZO, J. C. et al. Comparison of anchorage strength of bonded-in steel bars with epoxy resin, varying the superficial treatments and moisture after bonding, using Corymbia citriodora wood. Current Journal of Applied Science and Technology, v. 28, n. 5, p. 1-6, $2018 \mathrm{~b}$.

PIGOZZO, J. C. et al. Design and execution of wood-concrete deck bridge. Current Journal of Applied Science and Technology, v. 28, n. 3, p. 1-10, 2018 c.

Francisco Antonio Rocco Lahr

Departamento de Engenharia Civil | Universidade de São Paulo | Av. Trabalhador São Carlense, 400, Centro | São Carlos - SP - Brasil | CEP 13566-590 | Tel.: (16) 3373-9483 | E-mail: frocco@sc.usp.br

\section{Eduardo Chahud}

Escola de Engenharia | Universidade Federal de Minas Gerais | Av. Antônio Carlos, 6627, Sala 3316, Pampulha | Belo Horizonte - MG Brasil | CEP 31270-901 | Tel.: (31) 3409-1961 | E-mail: echahud@gmail.com

\section{Felipe Nascimento Arroyo}

Departamento de Engenharia Civil | Universidade Federal de São Carlos | Rodovia Washington Luís (SP-310), Km 235 | São Carlos - SP Brasil | CEP 13565-905 | E-mail: lipe.arroyo@gmail.com

\section{André Luis Christofro}

Departamento de Engenharia Civil | Universidade Federal de São Carlos | Tel.: (16) 3351-8262 | E-mail: christoforoal@yahoo.com.br

\section{Edson Fernando Castanheira Rodrigues}

Departamento de Engenharia Civil | Universidade Federal de São Carlos | E-mail: edson.engcivil.projetos@gmail.com

\section{João Paulo Boff Almeida}

Departamento de Engenharia Civil | Universidade Federal de São Carlos | E-mail: boff.joaopaulo@gmail.com

\section{Vinicius Borges de Moura Aquino}

Departamento de Engenharia Civil | Universidade Federal do Sul e Sudeste do Pará | Rua Geraldo Ramalho, s/n, Centro | Santana do Araguaia - PA - Brasil | CEP 68560-000 | Tel.: (94) 2101-5936 | E-mail: aquino.vini@hotmail.com

\section{Ambiente Construído}

Revista da Associação Nacional de Tecnologia do Ambiente Construído Av. Osvaldo Aranha, $99-3^{\circ}$ andar, Centro

Porto Alegre - RS - Brasil

$$
\text { CEP } 90035-190
$$

Telefone: +55 (51) 3308-4084

www.seer.ufrgs.br/ambienteconstruido www.scielo.br/ac

E-mail: ambienteconstruido@ufrgs.br 